\title{
Síndrome de Rett: análisis molecular del gen MECP2 en pacientes chilenas
}

\author{
Rett Syndrome: MECP2 gene molecular analysis in Chilean patients \\ Carolina Aron W. ${ }^{\text {a }}$, Geraldinne Rauch L. ${ }^{a}$, Felipe Benavides G. ${ }^{b}$ y M. Gabriela Repetto L. ${ }^{c}$ \\ aEstudiante de Medicina \\ bBioquímico \\ ‘Médico Genetista, Facultad de Medicina, Centro de Genética y Genómica, Clínica Alemana Universidad Del Desarrollo. Santiago, Chile
}

Recibido el 4 de mayo de 2018; aceptado el 5 de noviembre de 2018

\section{Resumen}

Introducción: El síndrome de Rett (RTT) es un trastorno neurológico progresivo caracterizado por producir una regresión del desarrollo psicomotor en niñas previamente sanas. La mayoría de los casos son causados por variantes patogénicas en el gen $M E C P 2$, que codifica para la proteína methyl CpGbinding protein 2. Objetivo: Describir la frecuencia y el tipo de variantes patogénicas en MECP2 en mujeres chilenas con diagnóstico clínico de RTT. Pacientes y Método: Se invitó a participar en este estudio a mujeres chilenas con sospecha clínica de RTT. Se reunió información clínica mediante un cuestionario. Se analizaron variantes patogénicas en MECP2 mediante el método de secuenciación de Sanger y se utilizó Multiple Ligation-dependant Probe Amplification (MLPA) para la detección de duplicaciones y deleciones. Resultado: El estudio incluyó 14 pacientes con sospecha de RTT, de las cuales $8(57 \%)$ pacientes tuvieron variantes patogénicas. Las restantes permanecen sin diagnóstico molecular. Conclusión: Variantes patogénicas en MECP2 están presentes en pacientes chilenas con RTT. Es probable que haya otros genes o diagnósticos involucrados en las pacientes sin hallazgos en MECP2. A partir de este trabajo, el diagnóstico molecular está disponible en Chile.

\section{Abstract}

Introduction: Rett syndrome (RTT) is a progressive neurological disorder characterized by regression of psychomotor development in previously healthy girls. Most cases are due to pathogenic variants in the MECP2 gene which encodes for the methyl CpG-binding protein 2. Objective: To describe the frequency and type of pathogenic variants in the MECP2 gene in Chilean female patients with clinical diagnosis of RTT. Patients and Method: Chilean women with clinical suspicion of RTT were invited to participate in the study. Clinical data were collected through a questionnaire. MECP2 pathogenic variants were analyzed by Sanger sequencing method and Multiplex Ligation-dependent
Palabras clave:

Síndrome de Rett; MECP2;

Proteína de unión a metil CpG2

Keywords:

Rett Syndrome;

MECP2;

Methyl-CpG-Binding

Protein 2 
Probe Amplification (MLPA) was used to detect duplications or deletions. Results: The study included 14 patients with suspected RTT, of which eight (57\%) patients had pathogenic variants. The other patients remain without molecular diagnosis. Conclusions: Pathogenic variants in MECP2 are present in Chilean patients with RTT. It is likely that there are other genes or diagnoses involved in patients without MECP2 findings. As of this study, molecular diagnosis is available in Chile.

\section{Introducción}

El síndrome de Rett (RTT; MIM\#312750) es un trastorno del neurodesarrollo que afecta principalmente a mujeres y es considerado prácticamente letal en hombres ${ }^{1}$. La incidencia se estima en 1 de cada 10.000 a 15.000 mujeres nacidas vivas ${ }^{2}$.

El diagnóstico de RTT se realiza en base a criterios clínicos $^{3}$ y existen tres formas clínicas: clásico o típico, variante o atípico y discapacidades de aprendizaje leve, siendo esta última poco frecuente. El RTT clásico es la forma más frecuente de presentación $(75 \%)^{4}$. Se caracteriza por un desarrollo aparentemente normal hasta los 6 a 18 meses de edad, seguido por una detención y posterior regresión en el desarrollo psicomotor, con pérdida de habilidades manuales, marcha, lenguaje y aparición de estereotipias. El RTT atípico tiene 5 posibles variantes: congénito, regresión tardía, lenguaje conservado, epilepsia precoz y forma incompleta, en las cuales pueden estar involucrados otros genes ${ }^{1-5}$.

Si bien el diagnóstico es clínico, el estudio molecular permite la confirmación. Variantes patogénicas en el gen MECP2 son la causa del $80-90 \%$ de los casos de RTT clásico y cerca del $40 \%$ de RTT atípico. La mayoría están ubicadas en los exones 3 y 4 , y el 99\% de las veces son de novo ${ }^{1-6}$. El resto de los casos de Rett se ha asociado a variantes patogénicas encontradas en los genes CDKL5 y FOXG1, las cuales se encuentran en mayor frecuencia en el Síndrome de Rett atípico ${ }^{7-8}$.

El gen $M E C P 2$ está ubicado en la región cromosómica Xq28, está compuesto por 4 exones que codifican para la proteína nuclear Methyl-CpG-binding protein $2{ }^{9}$. Esta proteína está involucrada en el desarrollo y maduración neuronal, así como también en la diferenciación y formación de sinapsis neuronales, mediante la regulación de la expresión de genes a través de metilación de islas $\mathrm{CpG}$, actuando tanto como represor y activador transcripcional ${ }^{10-12}$. La proteína tiene 4 dominios funcionales, de los cuales los más importantes son MBD (Methyl-CpG-binding Domain) y TRD (Transcription Repression Domain) ${ }^{1,11}$.

Las bases moleculares del síndrome de Rett en Chile no han sido caracterizadas. En esta investigación, se analizó el tipo y la frecuencia de variantes en el gen $M E C P 2$ en pacientes chilenas con diagnóstico clínico de RTT.

\section{Pacientes y Método}

Se invitó a participar, previo consentimiento informado de sus padres, a mujeres miembros de la Fundación Síndrome de Rett Chile el año 2014 con sospecha o diagnóstico clínico de RTT. El estudio fue aprobado por el Comité de Ética de Investigación del Centro de Bioética de la Facultad de Medicina Clínica Alemana Universidad del Desarrollo, de acuerdo a los términos de la declaración de Helsinki ${ }^{13}$.

La información clínica se obtuvo mediante un cuestionario con preguntas abiertas y cerradas, que incluía información acerca de antecedentes familiares, antecedentes perinatales y criterios diagnósticos de síndrome de Rett.

Para detectar variantes e implementar el diagnóstico molecular en el gen $M E C P 2$, se secuenció el gen utilizando partidores específicos, diseñados mediante el programa Primer3 para la región codificante exones $1,2,3$ y $4^{14}$. Los partidores obtenidos se verificaron mediante SNPCheck y BLAST ${ }^{15,16}$ y se añadió a cada partidor colas universales M13 para simplificar el procedimiento de secuenciación capilar ${ }^{17}$. Los exones fueron amplificados mediante un programa touchdown $68^{\circ}$ $58^{\circ} \mathrm{C}$ con 1 minuto de extensión, utilizando la enzima Mango Taq polimerasa (Bioline-UK). Los productos de PCR amplificados fueron secuenciados en ambos sentidos con BigDye 1.1 siguiendo el protocolo del fabricante (ThermoFischer-USA). Para la detección de deleciones o duplicaciones en MECP2 se utilizó Multiplex ligation-dependent probe amplification (MLPA) kit P245 (MRC-Holland) ${ }^{18}$.

\section{Resultados}

Participaron 15 mujeres entre 2 y 28 años, miembros de la Fundación Síndrome de Rett Chile. Una paciente fue excluida por no cumplir los criterios diagnósticos.

De las 14 pacientes, 10 tenían características de RTT clásico y 4 de RTT atípico. La información recolectada no permitió clasificar a estas últimas en los subtipos de RTT atípico. Las manifestaciones clínicas se resumen en la tabla 1.

Mediante secuenciación Sanger, se encontró 6 variantes patogénicas, 5 de ellas en pacientes con pre- 
Tabla 1. Características clínicas y moleculares de las pacientes

\begin{tabular}{|c|c|c|c|c|c|c|c|c|c|c|c|c|c|c|c|c|c|c|}
\hline \multirow{2}{*}{$\begin{array}{l}\text { Criterios } \\
\text { Pacientes }\end{array}$} & \multicolumn{4}{|c|}{ Criterios mayores } & \multicolumn{11}{|c|}{ Criterios menores } & \multicolumn{2}{|c|}{ Criterios exclusión } & \multirow{2}{*}{$\begin{array}{c}\text { Variante } \\
\text { patogénica }\end{array}$} \\
\hline & 1 & $\|$ & III & IV & 1 & 2 & 3 & 4 & 5 & 6 & 7 & 8 & 9 & 10 & 11 & $\mathrm{i}$ & ii & \\
\hline \multicolumn{19}{|c|}{ RTT Clásico } \\
\hline 1 & + & + & + & + & + & + & + & + & + & - & - & + & + & + & + & - & - & Glu37Argfs \\
\hline 2 & + & + & + & + & + & + & + & + & + & + & + & + & + & + & + & - & - & Thr158Met \\
\hline 3 & + & + & + & + & - & - & + & + & + & - & + & - & + & - & + & - & - & Gly269Alafs \\
\hline 4 & + & + & + & + & - & + & - & + & - & + & + & - & - & + & + & - & - & Arg270Ter \\
\hline 5 & + & + & + & + & - & + & + & + & - & - & + & + & + & + & + & - & - & Arg306Cys \\
\hline 6 & + & + & + & + & + & + & + & + & + & + & - & + & + & + & + & - & - & Del. exón 3 \\
\hline 7 & + & + & + & + & + & + & + & + & + & + & + & + & + & + & + & - & - & - \\
\hline 8 & + & + & + & + & + & - & + & + & + & + & + & + & + & - & + & - & - & - \\
\hline 9 & + & + & + & + & + & + & + & + & + & - & + & + & + & + & + & - & - & - \\
\hline 10 & + & + & + & + & + & + & + & + & + & - & + & + & + & + & + & - & - & - \\
\hline \multicolumn{19}{|c|}{ RTT Atípico } \\
\hline 11 & + & + & - & + & + & + & - & + & + & + & - & + & - & + & + & - & - & Arg106Trp \\
\hline 12 & + & + & - & + & - & + & + & - & + & + & + & + & + & + & + & - & - & Del. exón 3 \\
\hline 13 & + & - & - & + & + & + & - & - & - & - & - & - & + & + & + & - & + & - \\
\hline 14 & - & - & + & + & + & - & + & + & + & - & + & + & - & + & + & - & + & - \\
\hline $\begin{array}{l}\text { Criterios dia } \\
\text { da parcial o } \\
\text { menores: } 1 . \\
\text { vasomotor } p \\
\text { respuesta al } \\
\text { o infeccioso. }\end{array}$ & $\begin{array}{l}\text { féric } \\
\text { lor. }\end{array}$ & & uni & ciór & . & & & rec & nier & 8. & & & & eños & fríos & $\begin{array}{l}\text { a pren } \\
\text { ón de } \\
\text { t. Ton } \\
\text { 5. } 9 \text { LI } \\
\text { o a tra }\end{array}$ & $\begin{array}{l}\text { anual } p \\
\text { otipias } \\
\text { ular an } \\
\text { risa ina } \\
\text { rastorn }\end{array}$ & $\begin{array}{l}\text { positiva. II. Pér } \\
\text { nnuales. Criteri } \\
\text { mal. 5. Trastor } \\
\text { cuados. 10. Bz } \\
\text { neurometabóli }\end{array}$ \\
\hline
\end{tabular}

Tabla 2. Variantes patogénicas encontradas en gen MECP2

\begin{tabular}{ccccc}
\hline Paciente & Variante encontrada ADN & Consecuencia proteína* & Dominio afectado & Tipo de mutación \\
\hline 1 & c.108_111delAGAA & p.Glu37Argfs** & N-terminal & Cambio marco lectura \\
2 & c.473C $>$ T & p.Thr158Met & MBD & Sentido erróneo \\
3 & c.806delG & p.Gly269Alafs & TRD & Cambio marco lectura \\
4 & c.808C $>$ T & p.Arg270Ter & TRD & Sin sentido \\
5 & c.952C $>$ T & p.Arg306Cys & TRD & Sentido erróneo \\
6 & Deleción exón 3 & & MBD & Deleción \\
11 & c.316C>T & p.Arg106Trp & MBD & Sentido erróneo \\
12 & Deleción exón 3 & & MBD & Deleción \\
\hline
\end{tabular}

*Según dbSNP, ClinVar, RettBase. **Variante se encuentra en el exón 2.

sentación de RTT clásico y 1 en paciente considerada como RTT atípico. Cinco de estas variantes patogénicas estaban en los exones 3 y 4 , y una en el exón 2. En las 8 pacientes que no presentaron variantes puntuales, se realizó un análisis de deleciones y duplicaciones utilizando MLPA. Con esta técnica, se detectó una deleción del exón 3, variante patogénica, en dos pacientes adicionales: una con RTT clásico y una atípico. Además, se encontró 1 variante de significado incierto (VSI).
En las 5 pacientes restantes no se encontró variantes patogénicas (tabla 2).

\section{Discusión}

Este es el primer estudio molecular reportado en Chile con pacientes de RTT. Se encontraron variantes patogénicas en MECP2 en $57 \%$ de las pacientes estudiadas. 
En este grupo de participantes, la frecuencia de presentación de RTT clásico encontrada fue de alrededor de $70 \%$ (10 pacientes), similar a lo descrito en la literatura $(\sim 75 \%)^{4}$, de las cuales el $60 \%$ (6 pacientes) presentó variantes patogénicas de MECP2 y el 10\% (1 paciente) una variante de significado incierto (VSI). En el caso de los pacientes consideradas como RTT atípico, el 50\% presentó variantes patogénicas en el gen $M E C P 2$, lo que es similar a lo reportado en la literatu$\mathrm{ra}^{6}$. Si bien el número de casos para análisis fue bajo, tiene relación con la baja prevalencia de este síndrome en la población general. Además, no se caracterizó dirigidamente los subtipos de las pacientes con RTT atípico, sin embargo, dos de ellas presentan lenguaje conservado y retraso del desarrollo psicomotor precoz, por lo que podrían tener alguna de estas variantes clínicas.

De las 8 variantes patogénicas halladas, incluyendo las deleciones, 4 están entre las variantes más frecuentes descritas (Arg106Trp, Thr158Met, Arg270Ter y Arg306Cys $)^{1,19}$. A pesar de que las variantes patogénicas en MECP2 ocurren de novo, existen variantes más frecuentes, probablemente debido a la presencia de hot spots mutacionales en este gen ${ }^{20}$. Las deleciones que incluyen 1 o más exones se han observado tanto en pacientes con RTT clásico y atípico ${ }^{21}$. Esta evidencia sugiere que es importante utilizar MLPA en los casos en que no se han encontrado variantes puntuales en el gen $M E C P 2$. Un $87 \%$ de las variantes patogénicas encontradas están ubicadas en los dominios MBD y TRD, en los exones 3 y 4, tal como ha sido descrito en estudios previos ${ }^{22-23}$.

Se encontró una deleción de 36 nucleótidos in frame, c.1157_1192del36, clasificada como VSI. En este caso, el análisis de los padres en el diagnóstico molecular es importante para clasificar este tipo de variantes, pero no se ha realizado aún en esta familia ${ }^{24}$.

Este estudio es el primero en reportar y documentar variantes patogénicas en el gen MECP2 en pacientes con RTT en Chile. Conocer las variantes patogénicas específicas confirma el diagnóstico y podría permitir el desarrollo de futuras terapias moleculares. En las niñas en que no hubo hallazgos en $M E C P 2$, especialmente las 2 pacientes atípicas, sería interesante evaluar los otros genes relacionados con el diagnóstico de RTT, CDKL5 y FOXG1, así como también considerar diagnósticos diferenciales de RTT.

A partir de este trabajo se encuentra disponible el examen molecular para el gen MECP2 en Chile y por lo tanto, la certeza diagnóstica.

\section{Responsabilidades Éticas}

Protección de personas y animales: Los autores declaran que los procedimientos seguidos se conformaron a las normas éticas del comité de experimentación humana responsable y de acuerdo con la Asociación Médica Mundial y la Declaración de Helsinki.

Confidencialidad de los datos: Los autores declaran que han seguido los protocolos de su centro de trabajo sobre la publicación de datos de pacientes.

\section{Derecho a la privacidad y consentimiento informa-} do: Los autores han obtenido el consentimiento informado de los pacientes y/o sujetos referidos en el artículo. Este documento obra en poder del autor de correspondencia.

\section{Conflicto de intereses}

Los autores declaran no tener conflicto de intereses.

\section{Agradecimientos}

Queremos agradecer a la Fundación Síndrome de Rett Chile y a las familias de las pacientes que motivaron y participaron en esta investigación. También agradecer a Sra. María Luisa Guzmán, EU, y Srta. Valeria Tampe por su aporte en la realización de este trabajo.

\section{Referencias}

1. Christodoulou J., Ho G. MECP2-Related Disorders. GeneReviews ${ }^{\circledR} ; 1993-2016$ [updated 2012 Jun 28], https://www.ncbi. nlm.nih.gov/books/NBK1497/, última visita 12-12-2017.

2. Pantaleón G, Juvier T. Bases moleculares del Síndrome de Rett, una mirada actual. Rev Chil Pediatr. 2015;86:142-51.

3. Neul J, Kaufmann W, Glaze D, et al. Rett Syndrome: Revised Diagnostic Criteria and Nomenclature. Ann Neurol. 2010;68:944-50.
4. Hagberg B. Rett syndrome: clinical peculiarities and biological mysteries. Acta Paediatr. 1995;84:971-6.

5. Hagberg B., Gillberg C. Rett variants +/- rettoid phenotypes. MacKeith Press. 1993;40-60.

6. Leonard H, Cobb S, Downs J. Clinical and biological progress over 50 years in Rett syndrome. Nat Rev Neurol. 2017;13:3751.

7. Ariani F, Hayek G, Rondinella D, et al. FOXG1 is responsible for the congenital variant of Rett syndrome. Am J Hum Genet2008;83:89-93.
8. Fehr S, Wilson M, Downs J, et al. The CDKL5 disorder is an independent clinical entity associated with early-onset encephalopathy. Eur J Hum Genet. 2013;21:266-73.

9. Mnatzakanian G, Lohi H, Munteanu I, et al. A previously unidentified MECP2 open reading frame defines a new protein isoform relevant to Rett syndrome. Nat Genet 2004;36:339-41.

10. Tate P, Skarnes W, Bird A. The methylCpG binding protein MeCP2 is essential for embryonic development in the mouse. Nat Genet. 1996;12:205-8. 
11. Nan X, Ng H, Johnson C, et al. Transcriptional repression by the methylCpG-binding protein MeCP2 involves a histone deacetylase complex. Nature. 1998;393:386-9.

12. Chahrour M, Jung SY, Shaw C, et al. MeCP2, a key contributor to neurological disease, activates and represses transcription. Science. 2008;320:1224-9.

13. World Medical Association Inc. Declaration of Helsinki. Ethical principles for medical research involving human subjects. J Indian Med Assoc. 2009;107:403; www.wma.net, última visita 14-09-2017.

14. Untergasser A, Nijveen $\mathrm{H}$, Rao X, Bisseling T, Geurts R, Leunissen JA. Primer3Plus, an enhanced web interface to Primer3. Nucleic Acids Res. 2007;35:71-4.

15. Chuang L, Cheng Y, Yang C. Specific primer design for the polymerase chain reaction. Biotechnol Lett. 2013;35:1541-9.

16. Yu B, Zhang C. In silico PCR analysis. Methods Mol Biol. 2011;760:91-107.

17. Pont-Kingdon G, Gedge F, WooderchakDonahue W, et al. Design and analytical validation of clinical DNA sequencing assays. Arch Pathol Lab Med. 2012;136:41-6.

18. Yi Z, Wang S, Li L, et al. Diagnosis of MECP2 duplications syndrome with molecular genetic techniques. Zhonghua Er Ke Za Zhi. 2014;52:937-41.

19. Hadzsiev K, Polgar N, Bene J, et al. Analysis of Hungarian patients with Rett syndrome phenotype for MECP2, CDKL5 and FOXG1 gene mutations. J Hum Genet. 2001;56:183-7.

20. Wan M, Lee SS, Zhang X, et al. Rett syndrome and beyond: recurrent spontaneous and familial MECP2 mutations at $\mathrm{CpG}$ hotspots. Am J Hum Genet. 1999;65:1520-9.
21. Archer H, Whatley S, Evans J, et al. Gross rearrangements of the $M E C P 2$ gene are found in both classical and atypical Rett syndrome patients. J Med Genet. 2006;43:451-6.

22. Das DK, Raha S, Sanghavi D, Maitra A, Udani V. Spectrum of MECP2 gene mutations in a cohort of Indian patients with Rett syndrome: report of two novel mutations. Gene. 2013;515:78-83.

23. Zhang X, Bao X, Zhang J, et al. Molecular characteristics of Chinese patients with Rett syndrome. Eur J Med Genet. 2012;55:677-81.

24. Richards S, Aziz N, Bale S, et al. Standards and guidelines for the interpretation of sequence variants: a joint consensus recommendation of the American College of Medical Genetics and Genomics and the Association for Molecular Pathology. Genet Med 2005;17:405-23. 